\title{
Entrainment to an Auditory Signal: Is Attention Involved?
}

\author{
Richard Kunert \\ Max Planck Institute for Psycholinguistics, Nijmegen, \\ the Netherlands, and Radboud University
}

\author{
Suzanne R. Jongman \\ Max Planck Institute for Psycholinguistics, Nijmegen, \\ the Netherlands
}

\begin{abstract}
Many natural auditory signals, including music and language, change periodically. The effect of such auditory rhythms on the brain is unclear however. One widely held view, dynamic attending theory, proposes that the attentional system entrains to the rhythm and increases attention at moments of rhythmic salience. In support, 2 experiments reported here show reduced response times to visual letter strings shown at auditory rhythm peaks, compared with rhythm troughs. However, we argue that an account invoking the entrainment of general attention should further predict rhythm entrainment to also influence memory for visual stimuli. In 2 pseudoword memory experiments we find evidence against this prediction. Whether a pseudoword is shown during an auditory rhythm peak or not is irrelevant for its later recognition memory in silence. Other attention manipulations, dividing attention and focusing attention, did result in a memory effect. This raises doubts about the suggested attentional nature of rhythm entrainment. We interpret our findings as support for auditory rhythm perception being based on auditory-motor entrainment, not general attention entrainment.
\end{abstract}

Keywords: attention, entrainment, rhythm, memory, word memory

Supplemental materials: http://dx.doi.org/10.1037/xge0000246.supp

Many natural auditory signals change periodically, such as cricket chirps, bird song, and human speech and music. Many nonhuman animals have been found to use auditory periodicities to entrain their motor behavior to an underlying beat (Schachner, Brady, Pepperberg, \& Hauser, 2009). What is the effect of auditory temporal regularities on humans? One widely influential theory suggests that the fluctuations inherent in "attentional energy" are affected. According to this dynamic attending theory (DAT; Jones, Boltz, \& Kidd, 1982; Large \& Jones, 1999), rhythmic perceptual input leads to the optimal allocation of attention: attentional fluctuations peak at the right moment when important input is expected. These attentional dynamics are crucial for music (Jones \& Boltz, 1989) and speech comprehension (Pitt \& Samuel, 1990). In the present experiments we investigate whether an auditory rhythm indeed affects attention, an effect which should be visible on a variety of tasks. To foreshadow our results, we find an effect for

Richard Kunert, Max Planck Institute for Psycholinguistics, Nijmegen, the Netherlands, and Donders Institute for Brain, Cognition and Behaviour, Radboud University; Suzanne R. Jongman, Max Planck Institute for Psycholinguistics.

Both authors contributed equally (joint first authorship).

We thank Marlinde Zijl for conducting all experiments and Ani Patel and Liz Race for helpful comments on an earlier version of this article. The Max Planck Society funds Richard Kunert through a PhD grant; during the work described in this article Suzanne R. Jongman was funded through an International Max Planck Research School (IMPRS) PhD fellowship. This research was supported by the Max Planck Institute Nijmegen Interdisciplinary Innovation grant scheme.

Correspondence concerning this article should be addressed to Suzanne R. Jongman, Max Planck Institute for Psycholinguistics, PO Box 310, 6500 AH, Nijmegen, the Netherlands. E-mail: srjongman@gmail.com entrainment during immediate judgment tasks, but no effect for memory tasks. These findings suggest that auditory entrainment is actually quite limited. We propose that rhythmic entrainment may not affect general attention as suggested by the dominant interpretation of DAT, but instead results from auditory-motor entrainment.

The DAT has been very influential as evidenced by more than 800 citations for two key papers each (per a Google Scholar search in March 2016; Jones \& Boltz, 1989; Large \& Jones, 1999). One behavioral demonstration of auditory entrainment is reported by Bolger, Trost, \& Schön (2013). They asked participants to listen to a simple rhythm while performing a simple visual judgment task in which participants are asked to decide whether they see an " $x$ " or a "+" symbol. The rhythm consisted of a looped sequence of one high tone (at Metrical Position 1; MP1) and seven low tones (MP2-MP8), see Figure 1 black bars. According to DAT, participants should act faster and/or more accurately near the rhythmic (and thus attentional) peak (MP1 \& MP8) compared with trough (MP4), see Figure 1 top line. Indeed, response times were lower if a visual stimulus was presented during MP1 or MP8 compared with MP4, suggesting that the rhythm entrained internal attentional fluctuations. Thus, a typical finding in support of DAT links a peak in "attentional energy" to faster motor output.

This raises the question of what the "attentional energy" postulated by the DAT actually represents in terms of neurophysiology and/or psychology. Large and Jones (1999) remain vague in this respect, writing that high attentional energy "reflects the idea that "something is anticipated around this point in time"' (p. 130). "Attentional energy" has subsequently been interpreted as referring to general attention. As a result, the entrainment of such general attention as described by DAT was invoked to explain how accents in a task-irrelevant auditory stimulus can affect visual 


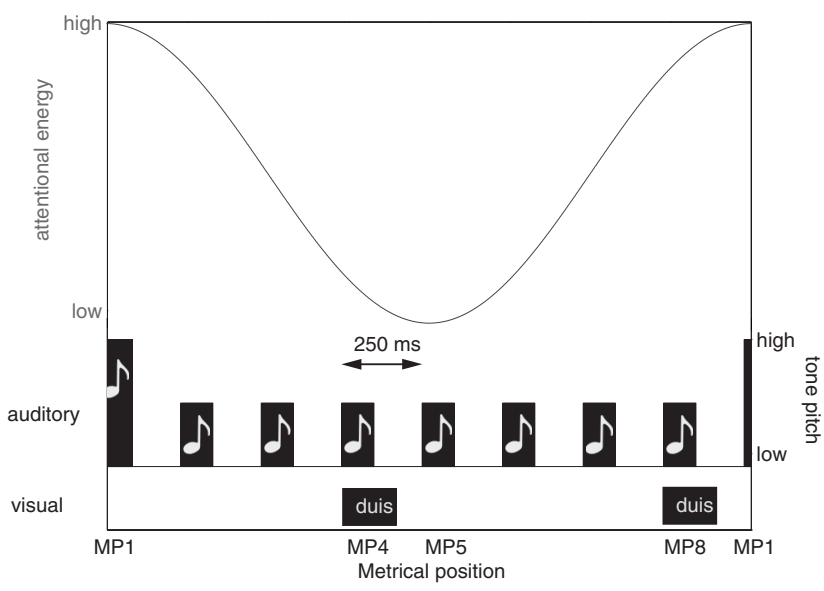

Figure 1. General procedure. The top line shows the prediction of dynamic attending theory (DAT): in this example participants' attentional energy (grey line) is entrained to a looped 2-s long sequence of one high and seven low tones (black middle bars) as described by Bolger et al. (2014). Presentation of visual targets (see rectangles at the bottom) occurs either near a rhythmic trough (Metrical Position 4; MP4) or just before a rhythmic peak (MP8).

(Bolger, Coull, \& Schön, 2014, 2013; Escoffier, Sheng, \& Schirmer, 2010; Escoffier \& Tillmann, 2008; Miller, Carlson, \& McAuley, 2013), auditory (Bolger et al., 2014, 2013), linguisticsyllabic (Brochard, Tassin, \& Zagar, 2013), and linguisticsemantic processing (Poulin-Charronnat, Bigand, Madurell, \& Peereman, 2005). Indeed, the obvious commonality shared by all these tasks appears to be that they depend on general attention. In this paper, we will adopt this dominant interpretation of the DAT as describing the entrainment of general attention to a perceptual rhythm.

Despite such a wide, supportive research literature, a growing number of research findings is not easily compatible with the DAT (Bauer, Jaeger, Thorne, Bendixen, \& Debener, 2015; Bermeitinger \& Frings, 2015; Hickok, Farahbod, \& Saberi, 2015; Kunert, Willems, \& Hagoort, 2016). This calls for a critical test of the theory. We believe that to prove that entrainment is due to general attention, the effect of entrainment should not only be present in tasks that require an immediate response, but it should also translate to secondary tasks, for instance to memory performance. Memory in general has been shown to be affected by general attention manipulations (Fernandes \& Moscovitch, 2000; Mulligan \& Hartman, 1996; Shtyrov, Kujala, \& Pulvermüller, 2010; Voss, Baym, \& Paller, 2008; see also Jacobson, Goren, Lavidor, \& Levy, 2012), and modulations of memory for simple auditory stimuli by a preceding rhythm has been invoked by Large and Jones (1999) as support for the DAT, rendering a visual memory task a good candidate to test whether rhythmic entrainment also affects general attention as predicted by the dominant interpretation of the DAT.

The DAT offers no neurophysiological account of how modulations of "attentional energy" through auditory entrainment affect cognition such as memory processes. We speculate that auditory entrainment could influence memory in a similar way to other attention manipulations. One attention manipulation that has been investigated previously using neuroimaging is selective attention.
Heightened attention for certain stimuli through selectivity could have a similar underlying mechanism as heightened attention for certain time points through entrainment. Uncapher and Rugg (2009) show that selective attention for either color or location features of objects results in better memory behaviorally, and neurally enhances cortical and hippocampal activity which is important for the consolidation of information from short-term memory (STM) to long-term memory. Optimal allocation of general attention via auditory entrainment might have the same effect. If so, a rhythmic peak should be associated with improved cortical processing in brain regions sensitive to the presented stimulus during encoding, leading to increased activity in the hippocampus, eventually resulting in better memory performance.

Testing this account behaviorally requires stimuli which evidence auditory entrainment, for example, in terms of the aforementioned typical reaction time (RT)-related entrainment effects in immediate responses. Therefore, in Experiment 1a we set out to conceptually replicate the finding that an auditory rhythm affects motor responses, this time using a lexical-decision task. Most previous studies used relatively simple perceptual judgment tasks, that is, deciding between two visual stimuli like " $x$ " and " + ". We first investigated whether rhythm entrainment also affects more complicated stimuli, that is, deciding whether a string of letters is a word or not. In Experiment $1 \mathrm{~b}$ a possible confound related to acoustic differences during stimulus processing was tested. Having evaluated whether our stimuli allow for auditory entrainment effects in general, we turned to a recognition memory task which is sensitive to attention in Experiments $2 \mathrm{a}$ and $2 \mathrm{~b}$. Will memory encoding be superior near a moment of rhythmic salience (alleged attentional peak) leading to better recognition memory, as predicted by a general attention account like DAT?

\section{Methods Common to Experiments 1a-2b}

\section{Analysis}

We report Bayesian analyses using Bayes factors (Dienes, 2008, 2014) which represent relative model support. As such, one needs to specify at least two models of which the data can support one more than the other. The null hypothesis simply predicts an effect size of 0 . The alternative hypothesis is based on previously re-

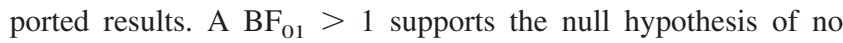
difference between conditions. $\mathrm{A} \mathrm{BF}_{10}>1$ supports the alternative hypothesis of a difference between conditions as predicted by previous literature. Jeffreys (1961) provides labels for interpretation: $1<\mathrm{BF}<3$ suggests that the evidence is not worth more than a bare mention, $3<\mathrm{BF}<10$ suggests substantial evidence for one model over the other, $10<\mathrm{BF}<30$ suggests strong and $30<$ $\mathrm{BF}<100$ very strong relative model support. A $\mathrm{BF}>100$ provides decisive evidence for one model over another. All priors for the alternative hypothesis were a normal distribution with $S D=$ mean/2. The chosen mean is reported for each experiment separately.

We also report analyses in the frequentist tradition for comparison (analysis of variance [ANOVA], $t$ test, 95\% confidence intervals [CIs]). In general, these results do not contradict the conclusions drawn from the Bayesian analyses. All analyses and figures can be reproduced with R-code and data provided in the online supplementary material. 


\section{Participants}

Each experiment sampled 60 different Dutch native speakers aged $M=21.7$ years (range $=18-38$ ) with little formal, musical training $(M=3.9$ years, range $=0-22)$ after obtaining their written, informed consent. Of the 240 participants overall, 207 were right-handed (range $=48-57$ per experiment), and 188 were female (range $=45-49$ per experiment). Given our use of Bayesian analyses, a formal power analysis in the frequentist tradition was not necessary (Wagenmakers, 2007). The chosen sample size of $N=60$ is greater than most other studies in this field, allowing for more evidence to decide between the null and the alternative hypothesis.

\section{Procedure}

Participants were tested in a dimly illuminated, soundproof booth, using a $17 \mathrm{in}$. screen and headphones. They read white letter strings shown in the middle of a black screen (font consolas, size 15) for a duration of $250 \mathrm{~ms}$. Time between letter string presentations was $2,000 \mathrm{~ms}$ to $7,000 \mathrm{~ms}$.

\section{Experiment 1a: A Regular Background Rhythm Influences Word Judgment Times}

\section{Introduction}

We first sought to conceptually replicate Bolger et al. (2013) by showing faster responses to visual targets presented near peaks in the auditory rhythm compared with troughs, as predicted by DAT. Bolger et al., like most researchers testing rhythmic entrainment, used a relatively easy visual judgment task with simple stimuli. Here we used a lexical-decision task where a string of letters was presented and participants judged whether the string was a word or not. It is important to test whether the DAT generalizes to a more difficult task and/or more variable stimuli. The need for such independent, conceptual replications has recently been underlined by replication problems in psychology in general (Kunert et al., 2016; Open Science Collaboration, 2015) and DAT research in particular (using a different paradigm, Bauer et al., 2015).

\section{Method}

Stimuli. A set of 144 monosyllabic words were matched to 144 monosyllabic pseudowords, created by WordGen (Duyck, Desmet, Verbeke, \& Brysbaert, 2004). All pseudowords were pronounceable and orthographically legal. Using the CELEX database, words and pseudowords were matched pairwise for length (3 to 5 letters, $M=4.2$ letters), and on average for neighborhood density (words: $M=6.95$; pseudowords: $\left.M=7.02 ; \mathrm{BF}_{01}=7.59\right)^{1}$ and bigram frequency (words: $M=23,971.22$; pseudowords: $M=$ $\left.21,800.90 ; \mathrm{BF}_{01}=3.71\right)$. Average word frequency was 33 tokens per million $(S D=66)$. The online supplementary material contains a complete stimulus list.

The rhythmic stimulus consisted of 8 tones of equal amplitude, starting with a high pitched tone at $880 \mathrm{~Hz}$ followed by seven low pitched tones at $440 \mathrm{~Hz}$. Each tone was presented for $100 \mathrm{~ms}$, with 250-ms SOA. One sequence therefore lasted $2 \mathrm{~s}$. Sequences were looped without pause in each block of trials. This auditory stimulus was the same as in Bolger et al. (2013), see Figure 1 middle bars for illustration.

Presentation of visual targets (see rectangles at the bottom of Figure 1) occurs either near a rhythmic trough (MP4) or near a rhythmic peak (MP8). Note that auditory stimulation is identical between MP4 and MP8.

Procedure. Participants were asked to judge whether a visually presented letter string was a legal Dutch word (50\% of trials) or not (50\% of trials) as quickly and accurately as possible by pressing either a right or a left button (button assignment counterbalanced across participants). There were six blocks of 48 trials each with uninterrupted, task-irrelevant auditory stimulation. Trial order was pseudorandomized such that each block contained an equal amount of words and pseudowords in each rhythm condition (MP4 = rhythmic trough and MP8 = rhythmic peak) with no more than 3 trials in each rhythm or lexicality condition after each other.

Analysis. To calculate the Bayes factor, not only the null hypothesis needs to be defined (an effect size of 0 ), but also the alternative hypothesis. This is based on previous findings. Regarding RTs, the alternative hypothesis predicts a 20-ms advantage for stimuli presented on or just before a rhythm peak (akin to MP8) compared with stimuli presented off peak (akin to MP4; Bolger et al., 2013; Escoffier et al., 2010). A similar 20-ms advantage was predicted for words over pseudowords based on a large scale Dutch visual lexical decision study (Keuleers, Diependaele, \& Brysbaert, 2010). No previous literature exists for the interaction between rhythmic entrainment and lexicality status. Therefore, we simply reasoned that if rhythm interacts with lexicality, we would expect that the MP8 condition enhances the processing of pseudowords more than the processing of words compared with the MP4 condition in which words are processed faster, possibly removing the entire lexicality effect (Garagnani, Shtyrov, \& Pulvermüller, 2009; Garagnani, Wennekers, \& Pulvermüller, 2008). Thus $20 \mathrm{~ms}$ was chosen as the mean for the alternative hypothesis prior of the interaction effect.

Regarding accuracy, the alternative hypothesis predicts a rhythm effect of $0.1 \%$ points based on Escoffier et al. (2010). Bolger et al. (2013) only reported that the difference for the two conditions did not reach significance without reporting mean values. Based on Keuleers et al. (2010), we expected a 10\%-point advantage for pseudowords over words. Finally, for the interaction we set the prior mean at $10 \%$ following the same reasoning as for the RT analysis.

For the frequentist analysis, repeated measures ANOVAs were performed.

\section{Results}

As predicted by the DAT, participants gave correct responses faster when the letter string was presented on MP8 $(M=606 \mathrm{~ms})$ than on MP4 ( $M=613 \mathrm{~ms}$, difference: $M=7.2 \mathrm{~ms}, 95 \%$ CI [3.3, $11.1], d=0.47)$; see Figure $2 \mathrm{~A}$. The data favor the alternative hypothesis of a difference between MP8 and MP4 $\mathrm{BF}_{10}=73.27$ times more than the null hypothesis of no difference, $F_{(1,59)}=$ 13.47, $p<.001, \eta_{G}^{2}=.003$. Furthermore, participants responded faster to real words $(M=585 \mathrm{~ms})$ than to pseudowords $(M=634 \mathrm{~ms}$,

\footnotetext{
${ }^{1}$ Bayes factors for stimulus matching calculated using JASP (Version 0.7.0) with a standard Cauchy prior (width $=0.707$ ).
} 
A) Exp. 1A: RT

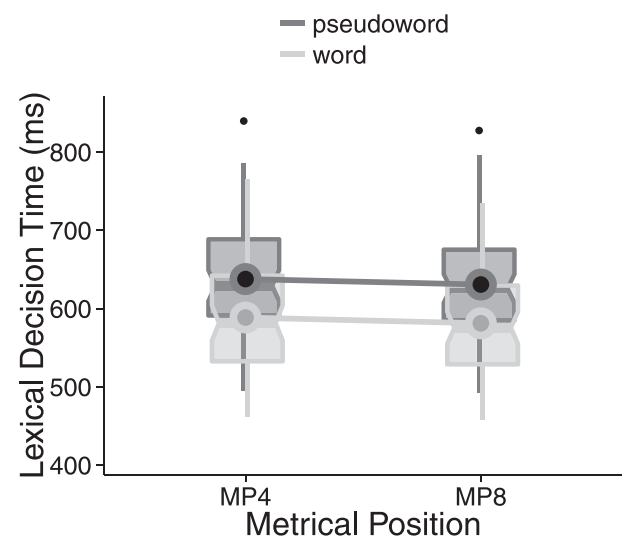

B) Exp. 1A: accuracy

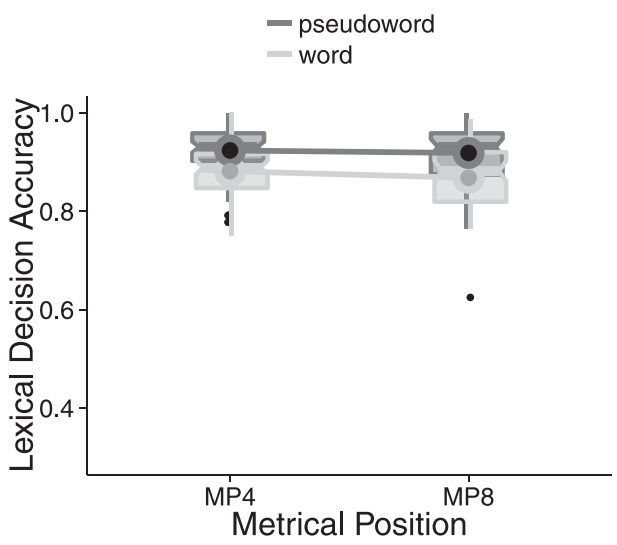

Figure 2. Results of Experiment 1a. Panel A: Reaction times (RT) for the lexical-decision task with a regular auditory rhythm in the background. RTs decrease near a moment of rhythmic salience (Metrical Position 8; MP8) compared with a less salient moment (MP4). Panel B: Accuracy levels for the lexical-decision task: no effect of the background auditory signal.

difference: $M=49 \mathrm{~ms}, 95 \% \mathrm{CI}[40,59], d=1.33)$, with decisive evidence for the alternative hypothesis, $\mathrm{BF}_{10}=1.25 \times 10^{21}$; $F_{(1,59)}=105.82, p<.001, \eta_{G}^{2}=.111$. Regarding the interaction, the null hypothesis was strongly supported, $\mathrm{BF}_{01}=18.65$, $F_{(1,59)}<1$.

There was no convincing evidence for a speed-accuracy tradeoff in the accuracy data as participants judged letter strings presented on MP4 minimally better $(M=90.3 \%)$ than on MP8 $(M=$ 89.3\%; difference: $M=-0.9,95 \%$ CI $[-1.7,-0.1], d=-0.29)$; see Figure 2B. The data minimally favor the alternative hypothesis, in other words there is no convincing evidence for either hypothesis, $\mathrm{BF}_{10}=1.72 ; F_{(1,59)}=5.08, p=.028, \eta_{G}^{2}=.006$. Pseudowords were responded to more accurately $(M=92.1 \%)$ than existing words $(M=87.5 \%$; difference: $M=4.6,95 \% \mathrm{CI}$ $[3.1,6.1], d=0.79)$, lexicality main effect with the alternative hypothesis decisively favored, $\mathrm{BF}_{10}=1.36 \times 10^{7} ; F_{(1,59)}=$ $37.79, p<.001, \eta_{G}^{2}=.136$. Rhythm and lexicality did not interact, $\mathrm{BF}_{01}=18.94 ; F_{(1,59)}=1.03, p=.313, \eta_{G}^{2}=.001$.

In sum, the results suggest that previously observed entrainment effects (Bolger et al., 2013) can be replicated with a lexicaldecision task, as predicted by DAT.

\section{Experiment 1b: An Irregular Background Signal Does Not Influence Word Judgments}

\section{Introduction}

Is an acoustic confound at the heart of the rhythm effect in Experiment 1a? Specifically, for MP8 the high tone in the series of low tones occurs just after the presentation of a visual target. Even though we argued MP4 and MP8 are acoustically identical, the auditory stimulation that follows it is not. Processing of the letter strings continues in time. Judgments are made around $600 \mathrm{~ms}$ after presentation onset at which point the following tone (a standard tone at MP5 and a high tone at MP1) has been heard. Therefore, the high tone after MP8 might act as a posttarget cue to attend, independent of its embedding in a regular rhythm (see Sergent et al., 2013). Experiment $1 \mathrm{~b}$ is a control experiment, identical to Experiment 1a except for the auditory signal which now includes unpredictably placed high tones. The DAT predicts a null effect.

\section{Method}

Stimuli. Compared with Experiment 1a, only the auditory signal differed. Eight different auditory stimuli contained seven low tones $(440 \mathrm{~Hz})$ and one high tone $(880 \mathrm{~Hz})$, each tone lasting $100 \mathrm{~ms}$ with an SOA of $250 \mathrm{~ms}$. In each stimulus, the high tone appeared at a different metrical position resulting in eight different rhythm sequences of $2 \mathrm{~s}$ each. During a block, as soon as one rhythm sequence had ended, a new one was randomly chosen out of the eight available and played once. There was no gap between stimuli. Visual targets were shown after at least two low tones and they appeared just before a low tone (like MP4 in Experiment 1a) or just before an unpredictable high tone (like MP8 in Experiment 1a).

Analysis. The analysis is the same as in experiment 1a.

\section{Results}

As predicted by the DAT and in contrast to Experiment 1a, letter strings presented before a high tone were responded to as fast (584 $\mathrm{ms})$ as those before a low tone $(582 \mathrm{~ms}$, difference: $M=-2.9 \mathrm{~ms}$, $95 \%$ CI $[-5.8,0.2], d=-0.25)$; see Figure $3 \mathrm{~A}$. There is substantial evidence for the null hypothesis, $\mathrm{BF}_{01}=4.70 ; F_{(1,59)}=$ $3.59, p=.063, \eta_{G}^{2}<.001$. Again, words were responded to faster $(M=562 \mathrm{~ms})$ than pseudowords $(M=604 \mathrm{~ms}$, difference: $M=$ $42 \mathrm{~ms}, 95 \%$ CI $[34,49], d=1.44)$, a lexicality effect with decisive support for the alternative, $\mathrm{BF}_{10}=5.87 \times 10^{25} ; F_{(1,59)}=124.90$, $p<.001, \eta_{G}^{2}=.091$. For the interaction, the null hypothesis was strongly preferred, $\mathrm{BF}_{01}=12.81 ; F_{(1,59)}<1$.

The accuracy data revealed no convincing evidence for a rhythm effect. Accuracy before a high tone was $89.5 \%$ compared with 
A) Exp. 1B: RT

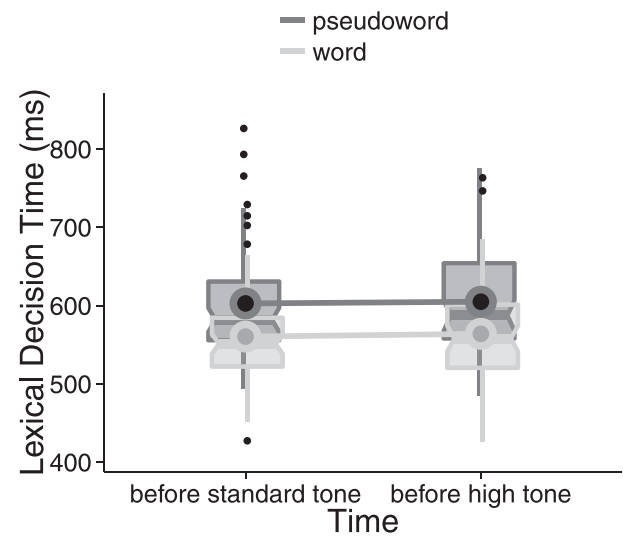

B) Exp. 1B: accuracy

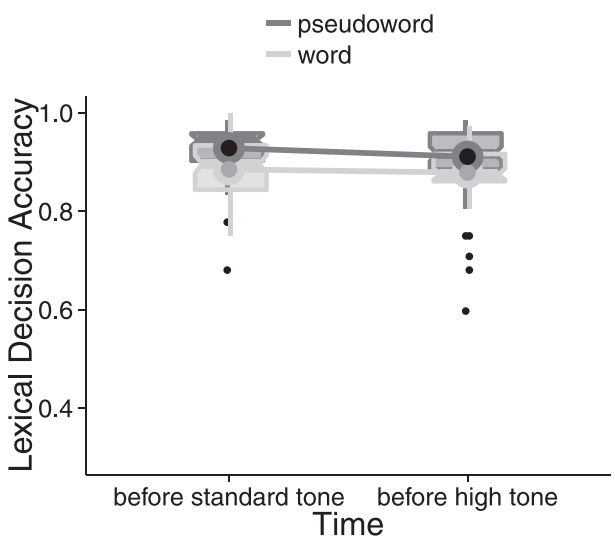

Figure 3. Results of Experiment 1b. Panel A: Reaction times (RT) for the lexical-decision task with an irregular auditory background signal. Strings presented before a standard tone (akin to Metrical Postion 4 [MP4] in Experiment 1a) are responded to as quickly as before a high tone (akin to MP8 in Experiment 1a). Panel B: Accuracy levels for the lexical-decision task. No convincing effect of the irregular auditory signal.

$90.7 \%$ before a low tone (difference: $M=-1.2 \%, 95 \% \mathrm{CI}$ $[-2.0,-0.4], d=-0.37)$, with minimal support for the alternative hypothesis, $\mathrm{BF}_{10}=1.99 ; F_{(1,59)}=8.05, p=.006, \eta_{G}^{2}=.010$; see Figure 3B. Just like in Experiment 1a, accuracy was lower for words $(88.2 \%)$ than for pseudowords $(92.0 \%$; difference: $M=$ $3.8 \%, 95 \%$ CI $[2.4,5.2], d=0.70)$, a lexicality main effect decisively favoring the alternative hypothesis, $\mathrm{BF}_{10}=1.51 \times 10^{5}$; $F_{(1,59)}=29.32, p<.001, \eta_{G}^{2}=.092$. There was no interaction, as the null hypothesis was strongly preferred, $\mathrm{BF}_{01}=12.97$; $F_{(1,59)}=1.23, p=.272, \eta_{G}^{2}=.002$.

In sum, the results suggest that an irregular auditory signal does not have the same effect as a regular one, excluding acoustic differences between MP4 and MP8 as a source of the RT effect in Experiment 1a.

\section{Experiment 2a: Rhythm During Pseudoword Encoding Is Without Influence on Later Recognition in Silence}

\section{Introduction}

The first set of experiments established that the chosen stimuli result in auditory entrainment as seen in immediate responses to letter strings. This validation of our stimuli allowed us to test the DAT's predictions on a second task. We asked participants to memorize pseudowords shown near a rhythmic peak (MP8) or trough (MP4). If rhythmic entrainment affects general attention, pseudowords shown on MP8 will be recognized better in a subsequent recognition memory test in silence, compared with those shown on MP4.

\section{Method}

Stimuli. The auditory stimulus was identical to Experiment 1a. The 144 pseudowords used in Experiment 1a and 1b acted as the recognition targets in the current experiment. Recognition foils were a new set of 144 pseudowords. Foils were created with WordGen (Duyck et al., 2004) and were pairwise matched for length ( 3 to 5 letters, $M=4.2$ letters), and on average matched for neighborhood density (targets: $M=7.02$; foils: $M=6.85$; $\mathrm{BF}_{01}=$ 7.24) and bigram frequency (targets: $M=21,800.90$; foils: $M=$ $\left.20,802.88 ; \mathrm{BF}_{01}=6.91\right)$ to the targets. An additional set of 24 pseudowords acted as fillers. All visual stimuli are provided in the online supplementary materials.

Procedure. Participants were asked to memorise pseudowords while hearing a background rhythm (encoding phase of the block), followed by judging whether a pseudoword was shown before (50\% of trials) or not (50\% of trials) without time pressure and in silence in the recognition phase of the block. There were six blocks. Each started with the passive encoding of pseudowords (shown near the rhythmic peak at MP8 or trough at MP4 for 250 ms) while hearing the looped regular rhythm (as in Experiment 1a). The encoding phase included first two fillers (avoid primacy effect), then 24 targets, and finally two fillers (avoid recency effect). The recognition of fillers was not tested. During the following recognition phase of the block, the same 24 target pseudowords were shown individually, intermixed with 24 foil pseudowords in a pseudorandom way (maximally 3 targets or foils after each other).

Analysis. Regarding the specification of the alternative hypothesis for the recognition memory performance, we based our expectations of the size of the rhythm effect on the effect of other attention manipulations on memory encoding, for example, full versus divided attention. Voss et al. (2008) tested the recognition memory of words previously shown under full attention or divided attention and found a full attention advantage in accuracy rates of approximately $10 \%$ points. This was taken as the prior mean of the rhythm effect. For recognition RT, the prior was set at $32 \mathrm{~ms}$ on the basis of a study by Craik, Govoni, Naveh-Benjamin, and Anderson (Experiment 4; 1996). In this experiment words previously presented under divided attention did not result in lower accuracy rates than fully attended words, but they were recognized slower. A dependent $t$ test was performed to test the influence of rhythm in the frequentist tradition. 


\section{Results}

The recognition in silence of pseudowords previously presented while hearing a regular rhythm showed no evidence for rhythm affecting encoding. Strings presented on MP8 were recognized as often $(76.6 \%)$ as strings presented on MP4 (77.1\%, difference: $M=-0.4,95 \% \mathrm{CI}[-2.4,1.5], d=-0.06), \mathrm{BF}_{01}=27.94 ; t_{(59)}<$ 1 , contradicting the DAT, see Figure 4A.

Given the surprising absence of auditory entrainment on recognition memory accuracy, we turn to the speed of recognition responses which has previously been linked to the confidence of memory judgments (Weidemann \& Kahana, 2016). Perhaps, RT provides a more fine grained measure than accuracy rates. However, the recognition RTs for pseudowords previously presented on MP8 $(M=704 \mathrm{~ms})$ were as high as those for strings previously presented on MP4 $(M=708 \mathrm{~ms}$, difference: $M=3.6 \mathrm{~ms}, 95 \% \mathrm{CI}$ $[-4.8,12.0], d=0.11), \mathrm{BF}_{01}=11.89 ; t_{(59)}<1$; see Figure 4B. To conclude, neither recognition accuracy nor recognition speed point to any influence of auditory entrainment during encoding.

\section{Experiment 2b: Even When Observing an Influence of Rhythm During Encoding There Is No Influence of Rhythm on Later Recognition}

\section{Introduction}

The lack of an effect of entrainment on recognition memory in Experiment 2a could have resulted from a lack of rhythmic entrainment during encoding. Did participants' focus on encoding for later recall prevent auditory entrainment? Task instructions can affect the neural entrainment to auditory rhythms (Iversen, Repp, \& Patel, 2009; Nozaradan, Peretz, Missal, \& Mouraux, 2011). Therefore, the task chosen in Experiment 2a might not have allowed for attentional entrainment. To test this possibility, Experiment $2 \mathrm{~b}$ used the same procedure as Experiment $2 \mathrm{a}$, but included an immediate vowel judgment task during the encoding phase in order to obtain a response time measure of entrainment.

\section{Method}

Stimuli. Both the visual and the auditory stimuli were identical to experiment $2 \mathrm{a}$.

Procedure. The experimental procedure was identical to Experiment $2 \mathrm{a}$, except that a task was added to the encoding phase. Participants were asked to indicate whether the pseudoword included the vowels $a$ and/or $i$ (49\% of trials) or not (51\% of trials) by pressing the right or left button (counterbalanced across participants) as fast and as accurately as possible.

Analysis. Regarding the vowel judgment during encoding, we chose the same priors as in Experiments 1a and 1b. For recognition memory, the Bayesian analysis was identical to Experiment 2a. The same prior as for the attentional effect on recognition memory was chosen for the $a / i$ versus non-a/i manipulation, both for recognition accuracy (10\%; Voss et al., 2008) and for recognition RT (32 ms; Craik et al., 1996), as we focused attention on the pseudowords containing the vowels $a$ and/or $i$ through the instructions. If rhythm interacts with vowel type, we would expect that the MP8 condition enhances processing of non-a/i pseudowords more than $a / i$-pseudowords, possibly removing the entire focused attention effect. The difference would remain intact in the MP4 condition. Thus $10 \%$ points was chosen as the mean for the alternative distribution. For recognition RT a prior of $32 \mathrm{~ms}$ was chosen for the interaction, following the same logic of the accuracy analysis.

For the frequentist analyses, we performed dependent ANOVAs.

\section{Results}

Regarding the vowel judgment during memory encoding in the context of an auditory rhythm, RTs on MP8 were lower $(M=582$ $\mathrm{ms})$ than on MP4 $(M=589 \mathrm{~ms}$, difference: $M=7.8 \mathrm{~ms}, 95 \% \mathrm{CI}$
A) Exp. 2A: recognition acc
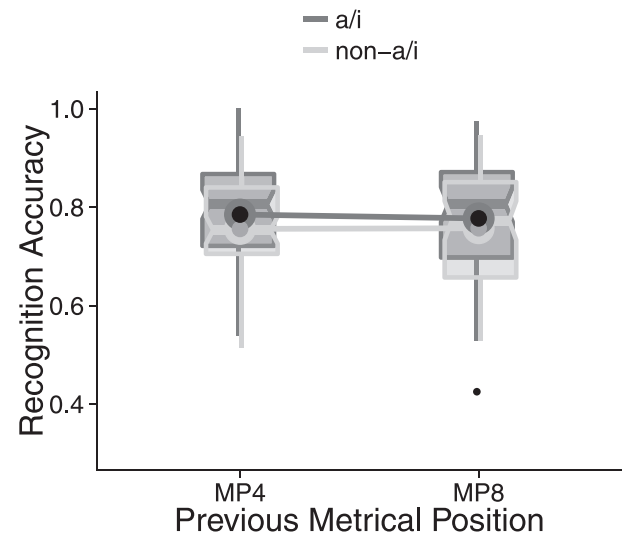

\section{B) Exp. 2A: recognition RT}

$-a / i$

- non-a/i

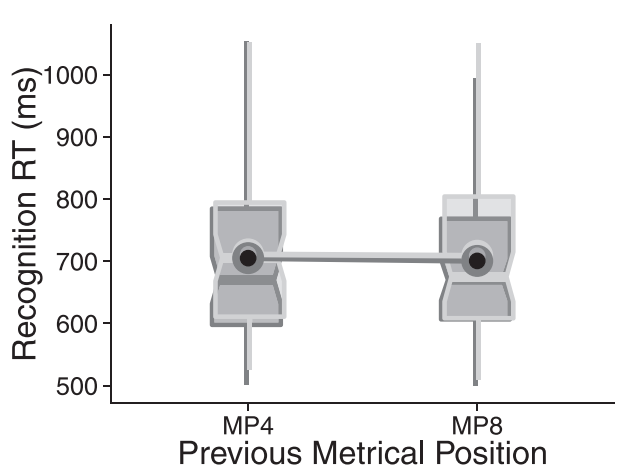

Figure 4. Results of Experiment 2a. Panel A: Recognition accuracy: a regular auditory background rhythm during pseudoword encoding does not influence accuracy levels for later recognition memory. Panel B: Recognition RT: a regular auditory background rhythm during pseudoword encoding does not affect the speed of correctly recognizing a pseudoword in a later memory task. 
$[3.3,12.3], d=0.44), \mathrm{BF}_{10}=40.13 ; F_{(1,59)}=11.84, p=.001$, $\eta_{G}^{2}=.005$, conceptually replicating the rhythm effect seen in Experiment 1a; see Figure 5A. Pseudowords with $a$ and/or $i$ were responded to faster $(M=566 \mathrm{~ms})$ than pseudowords including other vowels ( $M=605 \mathrm{~ms}$, difference: $M=39 \mathrm{~ms}, 95 \%$ CI [29.8, $47.8], d=1.12), \mathrm{BF}_{10}=1.58 \times 10^{15} ; F_{(1,59)}=74.73, p<.001$, $\eta_{G}^{2}=.107$. There was insufficient evidence to determine the presence of an interaction, $\mathrm{BF}_{01}=1.09 ; F_{(1,59)}=3.29, p=.075$, $\eta_{G}^{2}=.001$.

There was no convincing evidence for a speed-accuracy tradeoff related to the rhythm effect according to the accuracy data. Accuracy on MP8 (89.4\%) was similar to accuracy on MP4 (90.0\%; difference: $M=-0.6,95 \%$ CI [ $-1.8,0.5], d=-0.18)$, a rhythm main effect minimally favoring the alternative hypothesis, $\mathrm{BF}_{10}=1.19 ; F_{(1,59)}=1.22, p=.274, \eta_{G}^{2}=.002$; see Figure 5B. Accuracy for pseudowords including the vowels $a$ and/or $i$ was as high $(90.4 \%)$ as the accuracy for stimuli not including these vowels (89.1\%; difference: $M=1.3,95 \%$ CI [-0.2, 2.7], $d=$ 0.23 ), a vowel type main effect for which the null hypothesis is substantially favored, $\mathrm{BF}_{01}=6.35 ; F_{(1,59)}=3.19, p=.079, \eta_{G}^{2}=$ .010 . For the interaction, the null hypothesis is very strongly preferred, $\mathrm{BF}_{01}=31.69 ; F_{(1,59)}<1$.

Regarding the recognition memory task, the rhythm manipulation presented during encoding again did not influence later recognition memory accuracy in silence $\left(M_{\mathrm{MP} 8}=68.7 \%\right.$; $M_{\mathrm{MP} 4}=69.5 \%$, difference: $M=-0.9,95 \%$ CI $[-2.7,1.0]$, $d=-0.12), \mathrm{BF}_{01}=18.31 ; F_{(1,59)}<1$, contradicting the DAT; see Figure 5C. Pseudowords including $a$ and/or $i$ were better remembered $(M=74.2 \%)$ than non- $a / i$ pseudowords $(M=$ $64.0 \%$, difference: $M=10.2,95 \%$ CI [6.7, 13.7], $d=0.76)$, $\mathrm{BF}_{10}=9.51 \times 10^{6} ; F_{(1,59)}=34.36, p<.001, \eta_{G}^{2}=.118$. Regarding the interaction, the data were insensitive, $\mathrm{BF}_{01}=$ $1.31 ; F_{(1,59)}=3.11, p=.083, \eta_{G}^{2}=.004$.

For recognition speed, taken as a measure of recognition confidence (Weidemann \& Kahana, 2016), RTs did not differ for pseudowords previously presented on MP8 $(M=759 \mathrm{~ms})$ or MP4 $(M=760 \mathrm{~ms}$, difference: $M=1.0 \mathrm{~ms}, 95 \%$ CI [-10.9, 15.2], $d=$ $0.04)$. There is strong support for the null hypothesis, $\mathrm{BF}_{01}=$ $11.12 ; F_{(1,59)}<1$; see Figure 5D. Pseudowords including $a$ and/or $i$ were correctly recognized more quickly $(M=750 \mathrm{~ms})$ than non-ali pseudowords ( $M=774 \mathrm{~ms}$, difference: $M=23.3 \mathrm{~ms}, 95 \%$ CI $[6.6 ; 40.1], d=0.36), \mathrm{BF}_{10}=19.97 ; F_{(1,59)}=7.76, p=.007$, $\eta_{G}^{2}=.010$. Regarding the interaction, the data were insensitive, $\mathrm{BF}_{10}=1.53 ; F_{(1,59)}=2.44, p=.123, \eta_{G}^{2}=.001$.

In sum, even when rhythmic entrainment is visible during the encoding phase of a memory task, no rhythmic influence on pseudoword memory is found. There is a possibility that some individuals do show this effect, but that overall the rhythm effect does not reach significance because the effect is relatively small when it is present. Post hoc correlations were performed to look at individual differences in sensitivity to auditory entrainment. According to DAT, we should observe a correlation between the size of the auditory entrainment effect during encoding (measured as RT or accuracy) and the effect of auditory entrainment on later recognition memory accuracy. However, there is no evidence for such a relationship, neither for the auditory entrainment effect as measured in vowel judgment RT nor accuracy. For vowel judgment RT, Pearson's $r=.04(N=60,95 \%$ CI $[-.21, .29])$; see Figure $5 \mathrm{E}$. The Bayes factor of $\mathrm{BF}_{01}=3.40$ substantially favors the null hypothesis (a prior of $r=.3$ was chosen based on the expectation of a medium-sized correlation; $p=.739$ ). Similarly for vowel judgment accuracy, there is no convincing evidence for a correlation with the rhythm effect in recognition accuracy as Pearson's $r=.13(N=60,95 \%$ CI [ $-0.13,0.34])$; see Figure 5F. The Bayes factor of $\mathrm{BF}_{\mathrm{O} 1}=1.33$ minimally favors the null hypothesis $(p=.309)$.

\section{Comparison Between Experiments 2a and 2b: Evidence for Attention Influencing Pseudoword Memory}

\section{Introduction}

It can be argued that the memory task used in both Experiments $2 \mathrm{a}$ and $2 \mathrm{~b}$ is not sensitive to attention manipulations at all. If true, the lack of an auditory entrainment effect on the memory task is not surprising, as no manipulation of general attention would influence memory performance. To test the memory task's sensitivity to attention manipulations, we contrasted the memory performance of Experiment 2a with that of Experiment $2 b$ on two dimensions. First, the tasks differ in the amount of attention available for encoding. In Experiment $2 \mathrm{a}$ participants had the single task of encoding whereas in Experiment $2 \mathrm{~b}$ they had to combine encoding with the immediate vowel judgment task. In essence, the two experiments compare encoding under full attention to encoding under divided attention, which has been shown to influence memory performance in many previous studies (Fernandes \& Moscovitch, 2000; Mulligan \& Hartman, 1996; Shtyrov et al., 2010; Voss et al., 2008).

Second, the tasks differ in the focus of attention. In Experiment $2 \mathrm{a}$ there is a broad focus on the stimulus as a whole, whereas in Experiment $2 \mathrm{~b}$ there is an additional narrow focus on vowels (in particular the vowels $a$ and $i$ ) of the letter strings which is not relevant for the memory task itself but instead for the additional vowel judgment task. Focused attention can lead to enhanced memory performance as shown by Jacobson et al. (2012). If these attention manipulations, full attention versus divided attention and broad focus versus vowel focus, do lead to changes in memory performance in our memory task, we can conclude that the task is sensitive to general attention manipulations and thus a valid task to test the DAT.

Analysis. For the comparison between Experiments 2a and $2 \mathrm{~b}$, the divided attention prior was chosen just like for recognition memory, namely $10 \%$ points as the mean of the alternative hypothesis based on Voss et al. (2008). For the frequentist analysis, independent $t$ tests were performed.

\section{Results}

Recognition accuracy for strings presented previously under full attention in Experiment $2 \mathrm{a}(M=75.6 \%)$ was higher than under divided attention in Experiment $2 \mathrm{~b}(M=67.1 \%$, difference: $M=$ $8.5,95 \%$ CI $[5.8,11.2], d=0.81), \mathrm{BF}_{10}=9.95 \times 10^{7} ; t_{(118)}=$ $6.29, p<.001$. Furthermore, the difference between the recognition of $a / i$-pseudowords and non- $a / i$-pseudowords is larger when participants focus on $a / i$-pseudowords in Experiment $2 \mathrm{~b}(M=$ $10.2 \%$ points) than when they focus on all pseudowords equally in 
A) Exp. 2B: judgment RT
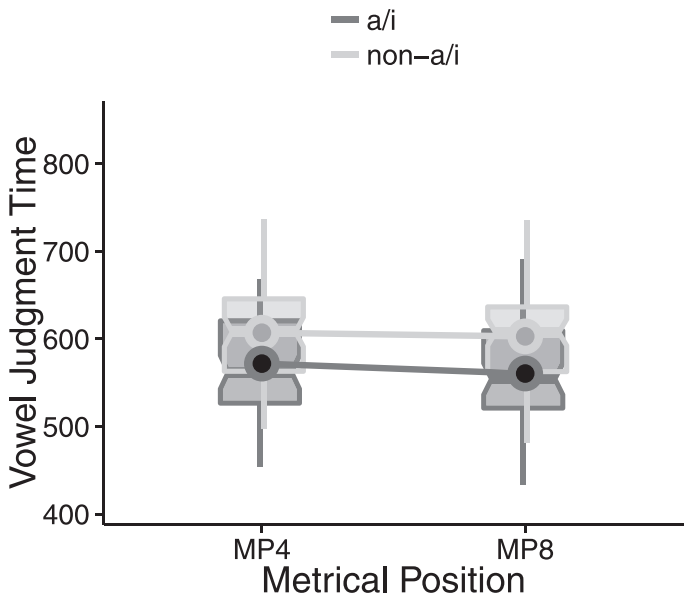

C) Exp. 2B: recognition acc

$$
\begin{aligned}
& -\mathrm{a} / \mathrm{i} \\
& -\mathrm{non}-\mathrm{a} / \mathrm{i}
\end{aligned}
$$

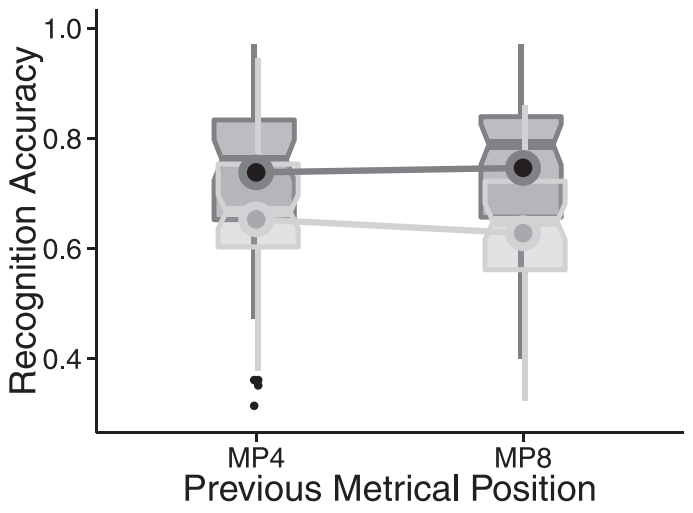

E) Exp. 2B: correlation RT

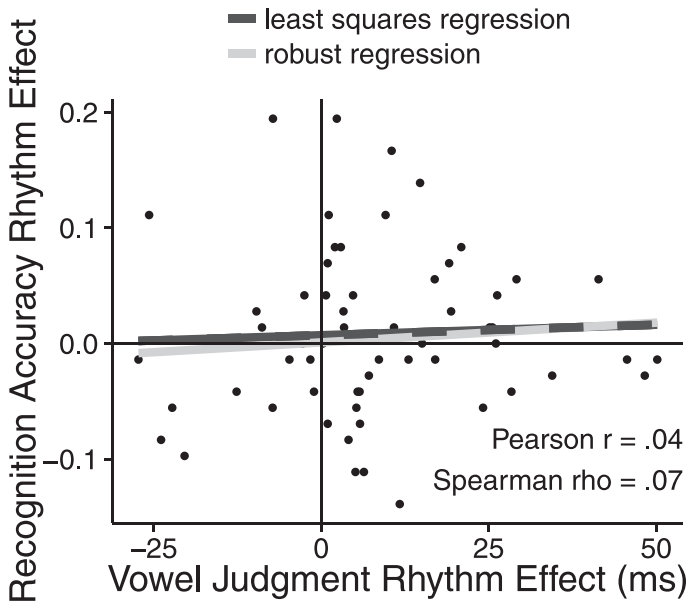

B) Exp. 2B: judgment accuracy

$-a / i$

- non-a/i

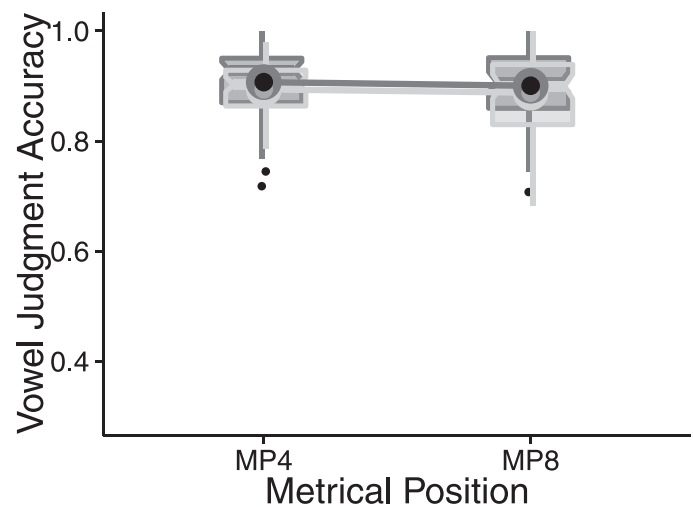

D) Exp. 2B: recognition RT

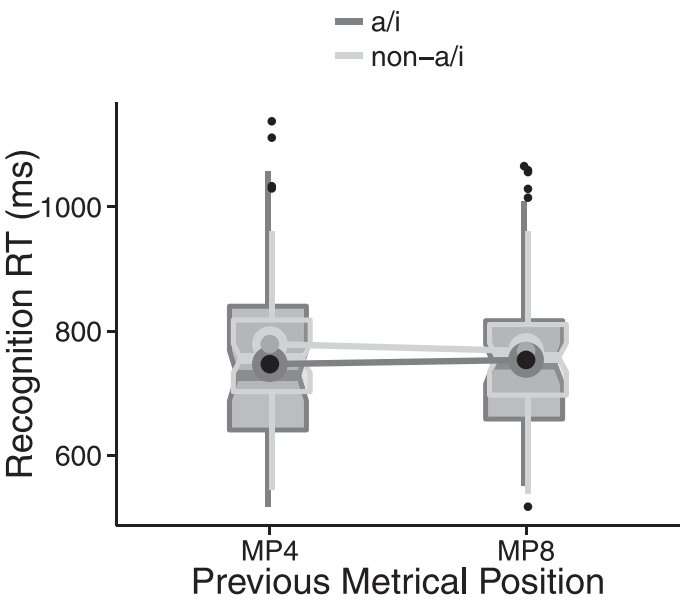

F) Exp. 2B: correlation acc

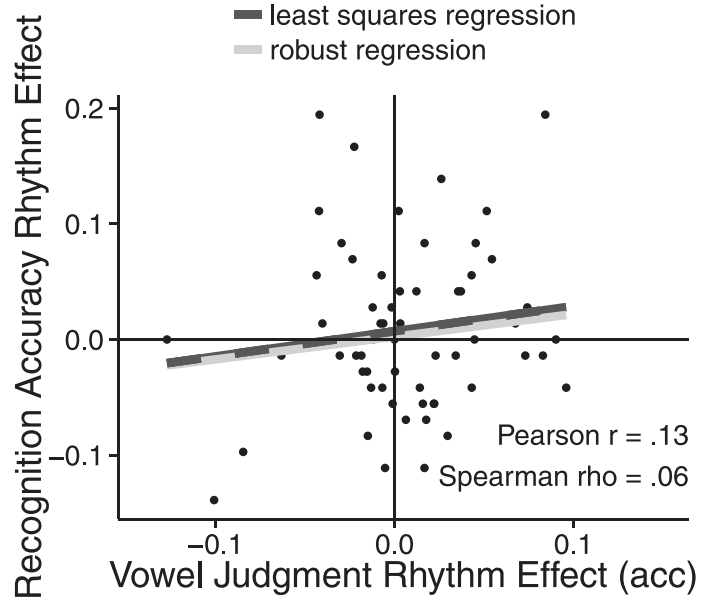


Experiment 2a $(M=2.5 \%$ points, difference: $M=7.7,95 \% \mathrm{CI}$ $[3.9,11.6], d=0.52), \mathrm{BF}_{10}=1,036.57 ; t_{(118)}=4.01, p<.001$.

Both results suggest that attentional manipulations can impact pseudoword learning. Recognition memory is better if encoding is performed under full attention (Figure 4A) compared with divided attention (Figure 5C). Moreover, the difference in recognition rates for strings including the vowels $a$ and/or $i$ and strings not including these vowels is smaller when this difference is irrelevant during encoding (i.e., broad focus, Figure 4A) compared with the situation in which vowels were relevant to the secondary vowel judgment task (i.e., narrow focus, Figure 5C). In sum, the comparisons between Experiment $2 \mathrm{a}$ and $2 \mathrm{~b}$ reveal that attention manipulations can in fact affect memory performance in our recognition task. The lack of a benefit in memory performance after rhythmic entrainment during encoding therefore cannot be explained by any insensitivity of our memory task to attention.

\section{General Discussion}

How does the brain react to rhythmic auditory input? Does it increase general attention at moments of rhythmic salience as predicted by the most-widely adopted interpretation of dynamic attending theory (DAT)? Experiment 1a replicated a typical response time effect associated with DAT: a lexical decision near a rhythmic peak is faster than near a rhythmic trough (see Bolger et al., 2013; Escoffier et al., 2010); see Figure 6A. A second prediction that we believe should follow from DAT, namely that the increase in general attention should improve encoding and in turn memory success, was not supported. Entrainment effects seen for immediate responses (Experiment 1a) do not extend to the encoding success of pseudowords (Experiments $2 \mathrm{a}$ and $2 \mathrm{~b}$ ), see Figure $6 \mathrm{~B}$. Showing a stimulus near a moment of rhythmic salience does not improve its later recognition.

Why did we not find any evidence for an attentional influence of auditory entrainment in Experiments $2 \mathrm{a}$ and $2 \mathrm{~b}$ ? One can rule out three confounds. First, auditory entrainment effects in RT (Experiment 1a) were not due to acoustic differences between rhythm conditions because an irregular auditory signal with the same acoustic properties was without effect in Experiment $1 \mathrm{~b}$; see Figure 6A. Second, the absent effect of entrainment on pseudoword memory in Experiment 2a was not due to the task demands precluding entrainment, given that in Experiment $2 b$ entrainment was evident in a secondary vowel judgment task performed during encoding. Third, the pseudoword memory task is not generally insensitive to attention manipulations (other than auditory entrainment) as evidenced by recognition differences due to attention demand differences between Experiments $2 \mathrm{a}$ and $2 \mathrm{~b}$, see Figure $6 \mathrm{C}$.

Still, a critical reader might argue that there are four different reasons for why the auditory entrainment effect on recognition memory is not evident in the data even though the DAT is true. We believe that none of these reasons provides a good account of our data. First, one could argue that the rhythm effects we find in the immediate judgment tasks in Experiment $1 \mathrm{a}$ and $2 \mathrm{~b}, 7$ and $8 \mathrm{~ms}$ respectively, are too small to translate to a memory effect. In order to evaluate this proposal we investigated individual differences in auditory entrainment effects in Experiment $2 b$. Are individuals with larger sensitivity to auditory entrainment in terms of immediate judgment RTs also more sensitive to auditory entrainment in terms of pseudoword encoding and subsequent memory performance? We find no evidence for this proposal $(r=.04)$. No correlation in terms of immediate judgment accuracy with memory performance was found either $(r=.13)$.

Second, one could argue that we merely found no effect due to lack of power, rather than evidence for the absence of an effect. However, the absent influence of auditory entrainment on recognition memory is not merely a nonsignificant result in the frequentist statistical tradition. Such frequentist results with a $p$ value above the significance threshold (usually $p>.05$ ) are indeed difficult to interpret as they might be due to low power (a false negative or type II error) or due to the genuine absence of an effect (a true negative). Instead, we provide clear results by performing a Bayesian analysis. It reveals strong evidence (Bayes factors $>10$ ) for the null effect of no influence of rhythm on memory in Experiments $2 \mathrm{a}$ and $2 \mathrm{~b}$; see Figure 6B. A low-powered design would presumably lead to an unclear Bayesian result supporting neither the null nor the alternative hypothesis (Bayes factors $<3$ ).

Third, one could argue that the expectation of finding a $10 \%$ point accuracy benefit for stimuli previously encoded under heightened attention (near rhythmic peak) compared with low attention (near rhythmic trough) is unrealistic given that the RTeffects of auditory entrainment in Experiments $1 \mathrm{a}$ and $2 \mathrm{~b}$ are already very small (7 to $8 \mathrm{~ms}$ ); see Figure 6A. Thus, would the Bayesian results change if the prior of the alternative hypothesis was changed to a smaller, more data-driven value? We turn to the effect of focused attention in Experiment $2 b$ in order to derive a novel expectation for the relation between RT-effects in immediate judgments and accuracy effects in recognition memory. An RT effect of $39 \mathrm{~ms}$ ( $a / i$ vs. non- $a / i$ judgment in Experiment 2b) translates into a memory effect of $10.2 \%$ points $(a / i$ vs. non- $a / i$

\footnotetext{
Figure 5 (opposite). Results of Experiment 2b. Panel A: Judgment RT: a regular auditory background rhythm during pseudoword encoding speeds up vowel judgment times near a moment of rhythmic salience (Metrical Postion 8; MP8) compared with a less salient moment (MP4). Panel B: Judgment accuracy: vowel judgment accuracy is not influenced by a background rhythm heard during encoding. Panel C: Recognition accuracy: a regular auditory background rhythm during pseudoword encoding does not influence accuracy levels for later recognition memory. Panel D: Recognition RT: response speed in the recognition memory task is not affected by a previously heard rhythm during encoding. Panel E: Correlation between vowel judgment RT rhythm effect and recognition accuracy rhythm effect: no relationship as evidenced by three correlation analyses (least squares regression, robust regression based on an iterated reweighted least squares regression which gives less weight to observations with large residuals compared with "typical" observations, and Spearman rho). Panel F: Correlation between vowel judgment accuracy rhythm effect and recognition accuracy rhythm effect: no relationship.
} 

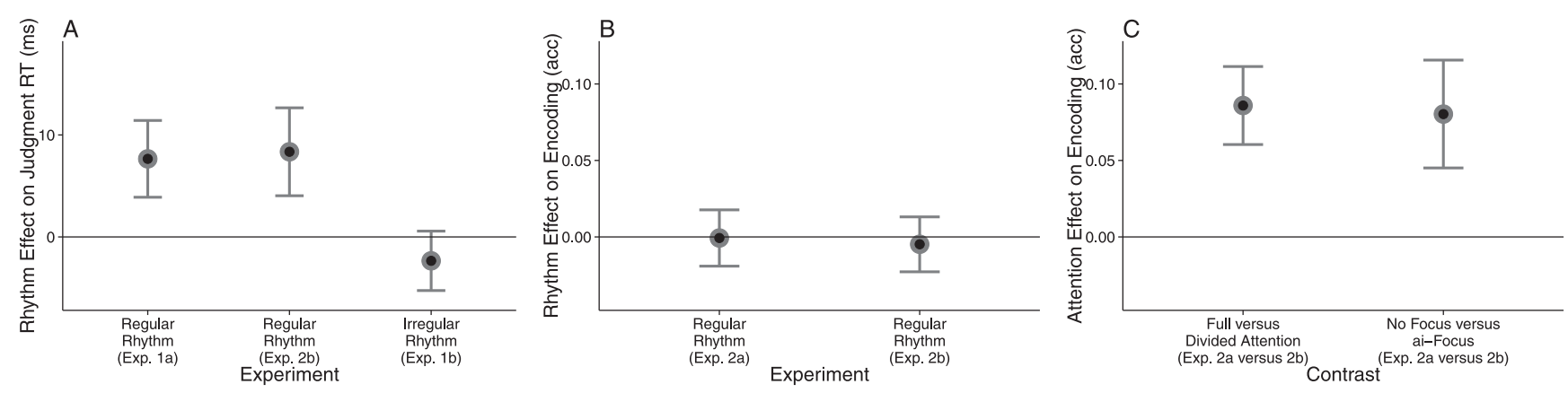

Figure 6. Summary of experimental results (posterior means and $95 \%$ credible intervals). Panel A: A regular rhythm affects word/vowel judgment speed while an irregular signal is without effect. Panel B: Rhythm does not affect pseudoword recognition memory. Panel C: Pseudoword recognition memory is better when encoding happened under full attention compared with divided attention. Moreover, focusing attention on $a / i$-pseudowords increases the difference in recognition performance between a/i-pseudowords and non-a/i-pseudowords compared with focusing attention on all pseudowords equally.

recognition memory effect in Experiment $2 b$ ). If the relation between an RT-effect in immediate judgments and an accuracy effect on later recognition is similar for auditory entrainment, then an effect of $7.8 \mathrm{~ms}$ (rhythm effect on vowel judgment speed in Experiment $2 b$ ), should have resulted in a later recognition effect of $2.04 \%$ points. Do the data support such a small rhythm effect on pseudoword memory more than the null hypothesis of no difference? No, a Bayesian analysis still does not point toward support for the alternative hypothesis of a rhythm effect on recognition memory accuracy of size $2.04 \%$ points (Experiment $2 \mathrm{a}: \mathrm{BF}_{01}=$ 2.73; Experiment $2 \mathrm{~b}: \mathrm{BF}_{\mathrm{O} 1}=1.43$ ). Note that neither Bayes factor is above 3 indicating that the support for the null hypothesis of no influence of the auditory signal on pseudoword memory is minimal for Experiments $2 \mathrm{a}$ and $2 \mathrm{~b}(\mathrm{BF}<3)$ and that more research is therefore warranted to more firmly exclude such a tiny recognition memory effect. Overall, this additional analysis shows that the effect of auditory entrainment on general attention might not just be very small. The data instead suggest that it could simply be absent.

Fourth, one might argue that intermingling high and low attention trials in the same block reduces the sensitivity of a subsequent memory test to reveal any influence of attention on encoding. The memory system could be said to be "too slow" to react to fast changes in attention. The difference in recognition rates between $a / i$-pseudowords and non- $a / i$-pseudowords in Experiment $2 \mathrm{~b}$ suggests that this is not the case; see Figure $6 \mathrm{C}$. Even though both kinds of pseudowords were intermingled and associated with different attentional states through a secondary vowel judgment task, the memory encoding system managed to adjust quickly to changing attentional states and revealed them later during recognition memory. Thus, the intermingling of supposedly low and high attention trials is probably not responsible for the absent influence of auditory entrainment on recognition memory.

Overall, the current results suggest that general attention is not involved in auditory entrainment, which we take to be problematic for the DAT framework. Although we cannot exclude the possibility that different task parameters (e.g., beatbased entrainment as in Miller et al. (2013) instead of meterbased entrainment here) might show entrainment effects influencing visual, linguistic, and memory processes via general attention, the DAT currently does not predict such task parameter influences.

An alternative model by Patel and Iversen (2014) better accounts for the limited effects of an auditory rhythm on the brain which we observed here. This model suggests that auditory entrainment is facilitated by a periodic, top-down signal from motor areas (see Large, Herrera, \& Velasco, 2015 for a computational model). Thus, auditory entrainment is limited to the auditorymotor axis, not extending to the visual, linguistic and memory systems engaged in the pseudoword memory task in Experiments $2 \mathrm{a}$ and $2 \mathrm{~b}$. This model is supported by neuroimaging evidence for motor system involvement during auditory rhythm perception in the absence of overt movement (Chen, Penhune, \& Zatorre, 2008a; Grahn \& Brett, 2007; Grahn \& Rowe, 2009). Moreover, interactions between motor and auditory regions can be observed during rhythm perception (Chen, Penhune, \& Zatorre, 2008b; Fujioka, Trainor, Large, \& Ross, 2012; Grahn \& Rowe, 2009). A limited auditory-motor account explains why entrainment is seen in audition (Hickok et al., 2015) and immediate motor output (Experiment 1a) but not on tasks relying on other cognitive systems (Experiments $2 \mathrm{a}$ and $2 \mathrm{~b}$ ).

In sum, our results suggest that entraining to an auditory rhythm only affects the motor and auditory systems, instead of extending via general attention to a wide variety of cognitive systems. Such a limited influence of auditory regularities is actually sufficient to explain everyday human experiences, like music rhythm facilitating dancing and clapping to the beat (motor system involvement) and speech rhythm facilitating language comprehension (auditory system involvement). Invoking general attention to explain entrainment effects is not necessary. This article suggests that rhythm entrainment amounts to auditory-motor entrainment, not attentional entrainment.

\section{References}

Bauer, A.-K. R., Jaeger, M., Thorne, J. D., Bendixen, A., \& Debener, S. (2015). The auditory dynamic attending theory revisited: A closer look at the pitch comparison task. Brain Research, 1626, 198-210. http://dx .doi.org/10.1016/j.brainres.2015.04.032 
Bermeitinger, C., \& Frings, C. (2015). Rhythm and attention: Does the beat position of a visual or auditory regular pulse modulate $\mathrm{T} 2$ detection in the attentional blink? Frontiers in Psychology: Cognition, 6, 1847. http://dx.doi.org/10.3389/fpsyg.2015.01847

Bolger, D., Coull, J. T., \& Schön, D. (2014). Metrical rhythm implicitly orients attention in time as indexed by improved target detection and left inferior parietal activation. Journal of Cognitive Neuroscience, 26, 593605. http://dx.doi.org/10.1162/jocn_a_00511

Bolger, D., Trost, W., \& Schön, D. (2013). Rhythm implicitly affects temporal orienting of attention across modalities. Acta Psychologica, 142, 238-244. http://dx.doi.org/10.1016/j.actpsy.2012.11.012

Brochard, R., Tassin, M., \& Zagar, D. (2013). Got rhythm . . . for better and for worse: Cross-modal effects of auditory rhythm on visual word recognition. Cognition, 127, 214-219. http://dx.doi.org/10.1016/j .cognition.2013.01.007

Chen, J. L., Penhune, V. B., \& Zatorre, R. J. (2008a). Listening to musical rhythms recruits motor regions of the brain. Cerebral Cortex, 18, 28442854. http://dx.doi.org/10.1093/cercor/bhn042

Chen, J. L., Penhune, V. B., \& Zatorre, R. J. (2008b). Moving on time: Brain network for auditory-motor synchronization is modulated by rhythm complexity and musical training. Journal of Cognitive Neuroscience, 20, 226-239. http://dx.doi.org/10.1162/jocn.2008.20018

Craik, F. I., Govoni, R., Naveh-Benjamin, M., \& Anderson, N. D. (1996). The effects of divided attention on encoding and retrieval processes in human memory. Journal of Experimental Psychology: General, 125, 159. http://dx.doi.org/10.1037/0096-3445.125.2.159

Dienes, Z. (2008). Understanding psychology as a science: An introduction to scientific and statistical inference. London, England: Palgrave Macmillan.

Dienes, Z. (2014). Using Bayes to get the most out of non-significant results. Frontiers in Psychology, 5, 781. http://dx.doi.org/10.3389/fpsyg .2014 .00781

Duyck, W., Desmet, T., Verbeke, L. P. C., \& Brysbaert, M. (2004). WordGen: A tool for word selection and nonword generation in Dutch, English, German, and French. Behavior Research Methods, Instruments, \& Computers, 36, 488-499. http://dx.doi.org/10.3758/BF03195595

Escoffier, N., Sheng, D. Y. J., \& Schirmer, A. (2010). Unattended musical beats enhance visual processing. Acta Psychologica, 135, 12-16. http:// dx.doi.org/10.1016/j.actpsy.2010.04.005

Escoffier, N., \& Tillmann, B. (2008). The tonal function of a taskirrelevant chord modulates speed of visual processing. Cognition, 107, 1070-1083. http://dx.doi.org/10.1016/j.cognition.2007.10.007

Fernandes, M. A., \& Moscovitch, M. (2000). Divided attention and memory: Evidence of substantial interference effects at retrieval and encoding. Journal of Experimental Psychology: General, 129, 155-176. http:// dx.doi.org/10.1037/0096-3445.129.2.155

Fujioka, T., Trainor, L. J., Large, E. W., \& Ross, B. (2012). Internalized timing of isochronous sounds is represented in neuromagnetic $\beta$ oscillations. The Journal of Neuroscience, 32, 1791-1802. http://dx.doi.org/ 10.1523/JNEUROSCI.4107-11.2012

Garagnani, M., Shtyrov, Y., \& Pulvermüller, F. (2009). Effects of attention on what is known and what is not: MEG evidence for functionally discrete memory circuits. Frontiers in Human Neuroscience, 3, 10. http://dx.doi.org/10.3389/neuro.09.010.2009

Garagnani, M., Wennekers, T., \& Pulvermüller, F. (2008). A neuroanatomically grounded Hebbian-learning model of attention-language interactions in the human brain. The European Journal of Neuroscience, 27, 492-513. http://dx.doi.org/10.1111/j.1460-9568.2008.06015.x

Grahn, J. A., \& Brett, M. (2007). Rhythm and beat perception in motor areas of the brain. Journal of Cognitive Neuroscience, 19, 893-906. http://dx.doi.org/10.1162/jocn.2007.19.5.893

Grahn, J. A., \& Rowe, J. B. (2009). Feeling the beat: Premotor and striatal interactions in musicians and nonmusicians during beat perception. The
Journal of Neuroscience, 29, 7540-7548. http://dx.doi.org/10.1523/ JNEUROSCI.2018-08.2009

Hickok, G., Farahbod, H., \& Saberi, K. (2015). The rhythm of perception: entrainment to acoustic rhythms induces subsequent perceptual oscillation. Psychological Science, 26, 1006-1013. http://dx.doi.org/10.1177/ 0956797615576533

Iversen, J. R., Repp, B. H., \& Patel, A. D. (2009). Top-down control of rhythm perception modulates early auditory responses. Annals of the New York Academy of Sciences, 1169, 58-73. http://dx.doi.org/10.1111/ j.1749-6632.2009.04579.x

Jacobson, L., Goren, N., Lavidor, M., \& Levy, D. A. (2012). Oppositional transcranial direct current stimulation (tDCS) of parietal substrates of attention during encoding modulates episodic memory. Brain Research, 1439, 66-72. http://dx.doi.org/10.1016/j.brainres.2011.12.036

Jeffreys, H. (1961). Theory of probability. New York, NY: Oxford University Press.

Jones, M. R., \& Boltz, M. (1989). Dynamic attending and responses to time. Psychological Review, 96, 459-491. http://dx.doi.org/10.1037/ 0033-295X.96.3.459

Jones, M. R., Boltz, M., \& Kidd, G. (1982). Controlled attending as a function of melodic and temporal context. Perception \& Psychophysics, 32, 211-218. http://dx.doi.org/10.3758/BF03206225

Keuleers, E., Diependaele, K., \& Brysbaert, M. (2010). Practice effects in large-scale visual word recognition studies: A lexical decision study on 14,000 Dutch mono- and disyllabic words and nonwords. Frontiers in Pscyhology, 1, 174. http://dx.doi.org/10.3389/fpsyg.2010.00174

Kunert, R., Willems, R. M., \& Hagoort, P. (2016). Language influences music harmony perception: Effects of shared syntactic integration resources beyond attention. Royal Society Open Science, 3, 150685. http:// dx.doi.org/10.1098/rsos.150685

Large, E. W., Herrera, J. A., \& Velasco, M. J. (2015). Neural networks for beat perception in musical rhythm. Frontiers in Systems Neuroscience, 9, 159. http://dx.doi.org/10.3389/fnsys.2015.00159

Large, E. W., \& Jones, M. R. (1999). The dynamics of attending: How people track time-varying events. Psychological Review, 106, 119-159. http://dx.doi.org/10.1037/0033-295X.106.1.119

Miller, J. E., Carlson, L. A., \& McAuley, J. D. (2013). When what you hear influences when you see: Listening to an auditory rhythm influences the temporal allocation of visual attention. Psychological Science, 24, 1118. http://dx.doi.org/10.1177/0956797612446707

Mulligan, N. W., \& Hartman, M. (1996). Divided attention and indirect memory tests. Memory \& Cognition, 24, 453-465. http://dx.doi.org/10 .3758/BF03200934

Nozaradan, S., Peretz, I., Missal, M., \& Mouraux, A. (2011). Tagging the neuronal entrainment to beat and meter. The Journal of Neuroscience, 31, 10234-10240. http://dx.doi.org/10.1523/JNEUROSCI.0411-11 .2011

Open Science Collaboration. (2015, August 28). Estimating the reproducibility of psychological science. Science, 349, aac4716. http://dx.doi.org/ 10.1126/science.aac4716

Patel, A. D., \& Iversen, J. R. (2014). The evolutionary neuroscience of musical beat perception: The action simulation for auditory prediction (ASAP) hypothesis. Frontiers in Systems Neuroscience, 8, 57. http://dx .doi.org/10.3389/fnsys.2014.00057

Pitt, M. A., \& Samuel, A. G. (1990). The use of rhythm in attending to speech. Journal of Experimental Psychology: Human Perception and Performance, 16, 564-573. http://dx.doi.org/10.1037/0096-1523.16.3 .564

Poulin-Charronnat, B., Bigand, E., Madurell, F., \& Peereman, R. (2005). Musical structure modulates semantic priming in vocal music. Cognition, 94, B67-B78. http://dx.doi.org/10.1016/j.cognition.2004.05.003

Schachner, A., Brady, T. F., Pepperberg, I. M., \& Hauser, M. D. (2009). Spontaneous motor entrainment to music in multiple vocal mimicking 
species. Current Biology, 19, 831-836. http://dx.doi.org/10.1016/j.cub 2009.03.061

Sergent, C., Wyart, V., Babo-Rebelo, M., Cohen, L., Naccache, L., \& Tallon-Baudry, C. (2013). Cueing attention after the stimulus is gone can retrospectively trigger conscious perception. Current Biology, 23, 150155. http://dx.doi.org/10.1016/j.cub.2012.11.047

Shtyrov, Y., Kujala, T., \& Pulvermüller, F. (2010). Interactions between language and attention systems: Early automatic lexical processing? Journal of Cognitive Neuroscience, 22, 1465-1478. http://dx.doi.org/10 $.1162 /$ jocn.2009.21292

Uncapher, M. R., \& Rugg, M. D. (2009). Selecting for memory? The influence of selective attention on the mnemonic binding of contextual information. The Journal of Neuroscience, 29, 8270-8279. http://dx.doi .org/10.1523/JNEUROSCI.1043-09.2009
Voss, J. L., Baym, C. L., \& Paller, K. A. (2008). Accurate forced-choice recognition without awareness of memory retrieval. Learning \& Memory, 15, 454-459. http://dx.doi.org/10.1101/lm.971208

Wagenmakers, E.-J. (2007). A practical solution to the pervasive problems of p values. Psychonomic Bulletin \& Review, 14, 779-804. http://dx.doi .org/10.3758/BF03194105

Weidemann, C. T., \& Kahana, M. J. (2016). Assessing recognition memory using confidence ratings and response times. Royal Society Open Science, 3, 150670. http://dx.doi.org/10.1098/rsos.150670

Received March 18, 2016 Revision received August 22, 2016 Accepted September 30, 2016

\section{ORDER FORM}

Start my 2017 subscription to the Journal of

Experimental Psychology: General ${ }^{\circledR}$ ISSN: 0096-3445

\section{$\$ 164.00$ APA MEMBER/AFFILIATE}

$\$ 411.00$ INDIVIDUAL NONMEMBER

$\$ 1,897.00$ INSTITUTION

Sales Tax: $5.75 \%$ in DC and $6 \%$ in MD and PA

TOTAL AMOUNT DUE

Subscription orders must be prepaid. Subscriptions are on a calendar year basis only. Allow 4-6 weeks for delivery of the first issue. Call for international subscription rates.

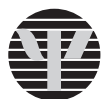

AMERICAN PSYCHOLOGICAL ASSOCIATION

\section{SEND THIS ORDER FORM TO}

American Psychological Association

Subscriptions

750 First Street, NE

Washington, DC 20002-4242

Call 800-374-2721 or 202-336-5600

Fax 202-336-5568 :TDD/TTY 202-336-6123

For subscription information,

e-mail: subscriptions@apa.org $\square$ Check enclosed (make payable to APA)

Charge my: $\square$ Visa $\square$ MasterCard $\square$ American Express

Cardholder Name

Card No. Exp. Date

Signature (Required for Charge)

\section{Billing Address}

Street

City State Zip

Daytime Phone

E-mail

Mail To

Name

Address

City State Zip 